\section{AB0601 RAPID AND SUSTAINED EFFICACY OF AN INDUCTION TREATMENT WITH A TRIPLE THERAPY INCLUDING HIGH-DOSE INTRAVENOUS IMMUNOGLOBULINS, METHOTREXATE AND GLUCOCORTICOIDS IN ANTI-3-HYDROXY-3-METHYLGLUTARYL-COENZYME A REDUCTASE MYOPATHY}

E. Treppo ${ }^{1}$, M. Infantino ${ }^{2}$, M. Benucci ${ }^{3}$, V. Ravagnani ${ }^{4}$, B. Palterer ${ }^{5}$, M. Grandis ${ }^{6}$, M. Fabris ${ }^{7}$, P. Tomietto ${ }^{8}$, M. Manfredi ${ }^{2}$, A. Sonaglia ${ }^{1}$, M. G. Giudizi ${ }^{5}$, F. Ligobbi ${ }^{3}$, D. Cammelli ${ }^{5}$, P. Parronchi ${ }^{5}$, S. De Vita ${ }^{1}$, L. Quartuccio ${ }^{1} .{ }^{1}$ Department of Medicine, Rheumatology Clinic, University of Udine, ASUFC Udine, Italy, Udine, Italy; ${ }^{2}$ Allergology and Immunology Laboratory Unit, Department of Laboratory Medicine, San Giovanni di Dio Hospital, Florence, Italy, Florence, Italy; ${ }^{3}$ Rheumatology Unit, Department of Medicine, San Giovanni di Dio Hospital, Florence, Italy, Florence, Italy; ${ }^{4}$ Rheumatology Unit, Mantova, Italy, Mantova, Italy; ${ }^{5}$ University of Florence, Florence, Italy; ${ }^{6}$ Department of Neuroscience (DINOGMI), San Martino Hospital, IRCCS, Genova, Italy, Genova, Italy; ${ }^{7}$ Department of Laboratory Medicine, ASUFC Udine, Udine, Italy, Udine, Italy; ${ }^{8}$ University of Trieste, Rheumatology Unit, Trieste, Italy

Background: Anti-3-hydroxy-3-methylglutaryl-coenzime A reductase (HMGCR) myopathy is a new entity, which has been clearly associated to statin use, even if it can be diagnosed in patients without a history of exposure to statin or even in the childhood (1).

Objectives: The aim of the study is to describe the efficacy of a triple therapy regimen consisting in high-doses of intravenous immunoglobulins (IVIG), methotrexate (MTX), and glucocorticoids (GC) in 16 patients with Anti-HMGCR myopathy enrolled in 6 specialized centres.

Methods: A total of 16 patients with anti-HMGCR myopathy ( 7 females; 9 males) were collected. Mean ( \pm standard deviation) age at the onset of disease was $72.4 \pm 10.3$ years old. All patients were diagnosed having anti-HMGCR myopathy [anti-HMGCR antibodies were measured by chemiluminescence assay (BioFlash, Inova, CA)] (2). Median follow-up was 29.5 months (interquartile range: 15.75-60 months). Anti-HMGCR antibodies were available in the follow-up in 8/16 patients.

Results: Thirteen out of 16 patients $(81.3 \%)$ had been exposed to statin (1/13 to red rice), $3 / 16$ (18.7\%) were not exposed. As induction therapy, 11/16 patients have been treated with triple therapy (high-dose IVIG, MTX and GC), 2/16 with double therapy (high-dose IVIG and GC), 2/16 have been treated with GC alone, the patient exposed to red rice resolved only with red rice suspension. Clinical remission and normalization of CPK values within month +24 were obtained in all the patients. All the patients were in remission at the last follow-up. Gradual improvement started soon from the first month, and among the 13 patients treated with an aggressive immunosuppresssive therapy including IVIG (13/13), GC (13/13) and methotrexate (11/13), 9/13 normalized the CPK value within 6 months. Clinical and laboratory response was accompanied by significant decrease or normalization of the antiHMGCR antibody titer. All the patients were either not taking GC $(56.3 \%)$, or were taking low doses of GC (43.7\%) at the last follow-up. Four patients had stopped GC within 6 months. No serious side effects were recorded. After persistent remission, a maintenance immunosuppressive therapy was then administered. Only 3 relapses in 3 different cases were recorded, all of them during drug-free remission in long-term follow-up. Reinduction was again effective in all.

Conclusion: Anti-HMGCR myopathy is a rare and serious myopathy which usually affects older people during statin treatment. After statin suspension, a rapid and sustained remission can be achieved by induction with a triple aggressive therapy consisting in medium-to high doses of GC, high-dose IVIG, and MTX (3). GC should be tapered as soon as possible. Relapse appears infrequent during maintenance treatment. Monitoring anti-HMGCR antibody titer may be clinically relevant.

References:

[1] AL Mammen et al. N Engl J Med. 2016;374:664-9

[2] Musset L et al. Autoimmun Rev. 2016;15:983-93.

[3] Aggarwal A et al. Scand J Rheumatol. 2019; 1-7.

Acknowledgments: We thank MD Francesca Grosso and MD Valentina Mecheri from the University of Florence, MD Angela Zuppa and MD Chiara De Michelis, from San Martino Hospital, Genova, for their valued collaboration in data collection

Disclosure of Interests: Elena Treppo: None declared, Maria Infantino: None declared, Maurizio Benucci: None declared, Viviana Ravagnani: None declared, Boaz Palterer: None declared, Marina Grandis: None declared, Martina Fabris: None declared, Paola Tomietto: None declared, Mariangela Manfredi: None declared, Arianna Sonaglia: None declared, Maria Grazia Giudizi: None declared, Francesca Ligobbi: None declared, Daniele Cammelli: None declared, Paola Parronchi: None declared, Salvatore De Vita Consultant of: Roche, GSK,
Speakers bureau: Roche, GSK, Novartis, Luca Quartuccio Consultant of: Abbvie, Bristol, Speakers bureau: Abbvie, Pfizer DOI: 10.1136/annrheumdis-2020-eular.2626

\begin{tabular}{l|l}
\hline AB0602 & SYSTEMIC SCLEROSIS: SUBCLINICAL \\
ATHEROSCLEROSIS AND MORBIMORTALITY.
\end{tabular}

M. Retuerto-Guerrero ${ }^{1}$, M. Martin Lopez ${ }^{1}$, B. Joven-Ibáñez ${ }^{1}$, J. L. Rosales ${ }^{2}$, P. Carreira ${ }^{1}{ }^{1}$ Hospital Universitario 12 de Octubre, Madrid, Spain; ${ }^{2}$ Hospiten Rambla, Santa Cruz de Tenerife, Spain

Background: Rheumatic diseases are associated with accelerated atherosclerosis, and an increase in cardiovascular morbidity and mortality. This process is mediated by classic cardiovascular risk factors (CVRF), chronic inflammation and atherogenic treatments such as corticosteroids. In Systemic Sclerosis (SSc) cardiovascular complications have increased in recent decades, although the studies on subclinical atherosclerosis (sATS) in SSc show discordant results.

Objectives: To evaluate prospectively the relationship between subclinical atherosclerosis, cardiovascular morbidity and mortality in patients with SSc.

Methods: 120 consecutive patients with SSc who attended their medical regula review during November and December 2011 were included. We evaluated the presence of plaques and measured the right CCA IMT by B doppler US for the detection of SATS (IMT> $0.9 \mathrm{~mm}$ and/or presence of plaque), review of classic CVRF and estimation of Medsger severity and EUSTAR activity index. Patients have been followed for 8 years, with at least annual consultation. In retrospect, the SCTC damage index, published in 2019, was obtained at the time of inclusion in the study. The clinical characteristics of the patients are collected since 1990 in a Prospective Longitudinal Observational Study (PLOS). Descriptive analysis was performed, using contigence tables for qualitative variables, and comparison of means for quantitative variables. The relationship between clinical characteristics, mortality, cardiovascular events (CVE), activity, severity and damage index, and SATS, was analyzed using binary logistic regression, adjusting for age and sex.

Results: 120 patients with SSc were included (93\% female, age $60 \pm 12$ years). 42 of these patients $(35 \%)$ had subclinical atherosclerosis. Age was statistically significant higher in patients with sATS compared to those without it $(67.9 \pm 11.5$ vs. $56.1 \pm 10.4$ years, $p<0.001$ ). We found no differences between groups in activity, damage and severity index (Table 1). Patients with sATS had higher levels of ESR and CRP, but the difference was not confirmed after adjusting for age $\left(^{*}\right)$. During the 8 years of follow-up, 9 CVE in 7 patients $(5.8 \%)$ : three myocardial infarction, one transient ischemic accident, one angor, one intermittent claudication and three refractory heart failure. The incidence of severe CVE was more than double in patients with SAtS $(10.25 \%$ vs. $3.7 \%)$, but the difference was not significant. We found no relationship between the mortality of any cause, or the secondary to CVE, with sATS, in the 32 patients who died during the follow-up, 3 due to CVE (9.4\%). The results are similar when we analyze only the presence of plaques.

Table 1. Relationship between clinical characteristics and activity, severity and damage index with the presence of accelerated atheromatosis.

\begin{tabular}{lccc}
\hline & Absent $\mathbf{n}=\mathbf{7 8}$ & Present $\mathbf{n}=\mathbf{4 2}$ & \\
\hline Diffuse cutaneous SSc & $37.18 \%$ & $26.12 \%$ & \\
mRSS & $7.71 \pm 6.32$ & $6.38 \pm 4.92$ & NS \\
High mRSS & $9.69 \pm 8.33$ & $7.57 \pm 4.74$ & $\mathrm{NS}$ \\
Arthritis & $32.05 \%$ & $28.57 \%$ & $\mathrm{NS}$ \\
Lung involvement & $33.33 \%$ & $30.95 \%$ & $\mathrm{NS}$ \\
PAH & $10.26 \%$ & $7.14 \%$ & $\mathrm{NS}$ \\
Cardiac involvement & $15.38 \%$ & $9.52 \%$ & $\mathrm{NS}$ \\
Digital ulcers & $35.90 \%$ & $35.83 \%$ & $\mathrm{NS}$ \\
AntiScl70 & $25.64 \%$ & $21.43 \%$ & $\mathrm{NS}$ \\
ESR & $20.12 \pm 13.77$ & $26.88 \pm 17.25$ & $\mathrm{p}=0.037^{*}$ \\
CPR & $0.65 \pm 0.61$ & $0.89 \pm 0.7$ & $\mathrm{p}=0.041^{*}$ \\
High activity index & $11.54 \%$ & $19.05 \%$ & $\mathrm{NS}$ \\
Damage index & $6.67 \pm 5.56$ & $5.62 \pm 4.9$ & $\mathrm{NS}$ \\
$\mathbf{\Sigma}$ Medsger index & $5.37 \pm 3.5$ & $5.05 \pm 3.45$ & $\mathrm{NS}$ \\
\hline
\end{tabular}

Conclusion: In our study subclinical atherosclerosis is not related to higher mortality in patients with SSc, but it does seem to influence the occurrence of cardiovascular events. In addition, our results suggest that SSc does not influence the onset of accelerated atheromatosis.

Acknowledgments: $M$ Retuerto was recipient of a training grant from Sociedad Española de Reumatologia (SER).

Disclosure of Interests: M. Retuerto-Guerrero: None declared, MARIA MARTIN LOPEZ: None declared, Beatriz Joven-lbáñez Speakers bureau: Abbvie Celgene, Janssen, Merck Sharp \& Dohme, Novartis, Pfizer, José Luis Rosales Grant/research support from: I have received financial support from Novartis, UCB, Pfizer, Abvie to meeting and symposia, Patricia Carreira Grant/research 
support from: Actelion, Roche, MSD, Consultant of: GlaxoSmithKline, VivaCell Biotechnology, Emerald Health Pharmaceuticals, Boehringer Ingelheim, Roche, Speakers bureau: Actelion, GlaxoSmithKline, Roche DOI: 10.1136/annrheumdis-2020-eular.6566

\section{AB0603 PDGFA AS A POTENTIAL BLOOD MARKER IN DSSC}

K. Romanowska-Prochnicka ${ }^{1,2}$, A. Wajda ${ }^{3}$, A. Paradowska-Gorycka ${ }^{3}$, A. FelisGiemza $^{2}$, E. Walczuk ${ }^{3}$, M. Olesińska ${ }^{2}$ on behalf of No. ${ }^{1}$ Medical University of Warsaw, Department of General and Experimental Pathology with Centre for Preclinical Research and Technology (CEPT), Warsaw, Poland; ${ }^{2}$ National Institute of Geriatrics, Rheumatology and Rehabilitation, Warsaw, Poland, Department of Systemic Connective Tissue Diseases, Warsaw, Poland; ${ }^{3}$ National Institute of Geriatrics, Rheumatology, and Rehabilitation, Department of Molecular Biology, Warsaw, Poland

Background: PDGF is a potential important factor in the pathogenesis of scleroderma. PDGF is almost undetectable in healthy skin or lung. Immunohistochemical studies have revealed increased presence of PDGF $\alpha$ and PDGF $\beta$ receptors in scleroderma skin biopsies.

Objectives: The aim of this study was to determine the mRNA level of IFNa1, $I L-4, T G F \beta 1, T G F \beta 2, P D G F a, P D G F \beta, T N F a$ in whole blood in SSc patients in the aspect of clinical

Methods: A group of 14 patients (50\% were women) with systemic sclerosis based on EULAR / ACR 2013 criteria was included in the study. The modified Rodnan Skin Score (mRSS) was evaluated by same assistant at the beginning of the study and six months later. DLCO, HRCT, echocardiography and NFC were measured. Gene expression was determined using validated TaqMan probes in qPCR. Constitutive mRNA level of selected genes was analyzed using $\Delta \mathrm{Ct}$ method. Comparison between different groups of patients was determined using non-parametric Mann-Whitney $U$ test. Correlation was analyzed using non-parametric Spearman test. Results: The mean age of the patients was $60 \pm 15.66 .100 \%$ of patients had organ involvement as pulmonary fibrosis. $78 \%$ - had active changes -features of ground glass.

$64 \%$ of patients had mild mRSS-1-10 skin involvement, $36 \%$ had moderate to severe skin involvement. In SSc patients TGF 1 and IFNa1 revealed the highest level of expression in comparison to other analyzed genes. Additionally, very high and significant correlation between TNF $a$ and TGF $\beta 1(r=0.7 \mathrm{p}=0.004)$ has been noted. High and significant correlation between mRNA PDGF $\beta$ and TNFa levels have been observed. We did not reveal significant differences in analyzed genes expression when compare limited and diffuse SSc. Nevertheless, patients with dSSc were characterized by higher level of IFNa1 (almost 2 times) and TGF $\beta 1$. On the border of significance higher PDGFa mRNA level was observed in dSSc patients when compared to ISSc. Average PDGFa expression is higher in SSc patients with Scl70 positive than than in patients without Scl70 $(p=0.04)$.

In the aspect of clinical parameters, patients with ESR $\leq 12 \mathrm{~mm} / \mathrm{h}$ revealed almost 6 times higher level of IFNa1 $(\mathrm{p}=0.01)$ in comparison to the patient with $E S R>12 \mathrm{~mm} / \mathrm{h}$. Patients with mRSS above10 points revealed significantly higher of PDGFa expression in comparison to patients with $\mathrm{mRSS} \leq 10(p=0.04)$. In these group of patients CRP and ESR were not different significantly.

In the case of patients with active fibrosis (ground glass) in HRCT IFNa1 expression was almost 2.5-times higher than in patients with HRCT non-active. Significantly higher PDGFa has been revealed in patients with active HRCT when compared with patient with non-active HRCT. Nevertheless these two groups did not differ in ESR or OB parameter.

SSc patients in active phase of NFC revealed almost 3 times higher level of IFNa1 expression in comparison with the patient in late phase.

Conclusion: The mRNA level of PDGFa may be a potential blood marker to predict worse prognosis in scleroderma

References:

[1] Rheumatology (Oxford). 2008 Oct;47 Suppl 5:v2-4. doi: 10.1093/ rheumatology/ken265.

Role of PDGF in fibrotic diseases and systemic sclerosis.

Trojanowska M1.

Acknowledgments: No

Disclosure of Interests: None declared

DOI: 10.1136/annrheumdis-2020-eular.5590

\section{$\mathrm{AB} 0604$ \\ EVALUATION OF PATIENTS WITH ANTISYNTHETASE SYNDROME AND INTERSTITIAL LUNG DISEASE BASED ON THE RADIOLOGICAL PATTERN}

A. Ruiz Roman ${ }^{1}$, J. A. Rodriguez Portal' . 'Hospital Virgen del Rocío, Rheumatology, Seville, Spain; ${ }^{2}$ Hospital Virgen del Rocío, Pneumology, Seville, Spain

Background: Antisynthetase syndrome is an autoimmune rheumatologic disease characterized by the presence of specific antibodies that are known as antisynthetase antibodies and with a varied clinic, including arthritis, myositis or interstitial lung disease (ILD), among others. ILD is the manifestation that associates the highest morbi / mortality and can occur with different characteristic radiological patterns: usual interstitial pneumonia (UIP), non-specific interstitial pneumonia (NSIP), organized pneumonia (OP)

Objectives: Describe the clinical, serological and prognostic characteristics of patients with antisynthetase syndrome with interstitial pulmonary involvement (ILD) based on their radiological pattern.

Methods: Retrospective descriptive study of patients with a clinical diagnosis of antisynthetase syndrome in which they show positive for some antisynthetase antibody and with clinical follow-up of at least 6 months in a reference center consultation between the period from January 2008 to September 2019. In those patients who presented interstitial lung involvement clinical, analytical and prognostic variables were evaluated (including spirometry) based on the radiological pattern presented by high-resolution computed tomography (HRCT) of the chest Results: 32 patients ( 24 women and 8 men) were included in the study. 7 cases $(21.9 \%)$ did not present pulmonary involvement, while the remaining 25 cases (78.1\%) presented with interstitial lung involvement. Of the patients diagnosed with ILD, 4 cases $(12.5 \%)$ had an interstitial pattern UIP, 17 cases $(53.1 \%)$ had an interstitial pattern of NSIP and another $4(12.5 \%)$ interstitial pattern of OP. From an analytical point of view, the most frequent antisynthetase antibody in our sample was antiJo1 with 29 cases (96.9\%) and less frequently the antiPL12 antibody with 2 cases and antiPL7 with 1 case. The specific characteristics depending on the type of EPID pattern are summarized in Table 1.

Table 1.

\begin{tabular}{|c|c|c|c|}
\hline \multirow[t]{2}{*}{ CHARACTERISTICS } & \multicolumn{3}{|c|}{ ILD PATTERN } \\
\hline & $\begin{array}{l}\text { Pattern UIP } \\
\quad \mathrm{N}=4\end{array}$ & $\begin{array}{c}\text { Pattern NSIP } \\
\quad \mathrm{N}=17\end{array}$ & $\begin{array}{l}\text { Pattern OP } \\
\quad \mathrm{N}=4\end{array}$ \\
\hline Gender (Female) n (\%) & $4(100)$ & $11(64.7)$ & $3(75.0)$ \\
\hline Age at diagnosis & $47.5 \pm 12.9$ & $52.9 \pm 10.5$ & $42.0 \pm 12.9$ \\
\hline Myositis, n(\%) & $2(50.0)$ & $13(76.5)$ & $4(100)$ \\
\hline Arthritis, n (\%) & $3(75.0)$ & 15 (88.2) & $3(75.0)$ \\
\hline Fever, n (\%) & $1(25.0)$ & $9(52.9)$ & $2(50.0)$ \\
\hline Mechanic's hands, n(\%) & $2(50.0)$ & $7(41.2)$ & $2(50.0)$ \\
\hline Raynaud's phenomen, $n$ (\%) & $1(25.0)$ & $8(47.1)$ & $1(25.0)$ \\
\hline Skin involvement, $n(\%)$ & $2(50.0)$ & $10(58.8)$ & $4(100)$ \\
\hline \multicolumn{4}{|l|}{ Treatment, $n(\%)$} \\
\hline Glucocorticoids & $4(100)$ & $16(94.1)$ & $4(100)$ \\
\hline Immunosuppresants & $4(100)$ & $16(94.1)$ & $4(100)$ \\
\hline Biological therapy & $1(25.0)$ & $8(47.1)$ & $2(50.0)$ \\
\hline $\begin{array}{l}\text { Drug's number (mean, SD) } \\
\text { Antisynthetase antibodies, } n\end{array}$ & $3 \pm 0.8$ & $5 \pm 2.9$ & $4.7 \pm 1.8$ \\
\hline Anti Jo1 & $3(75.0)$ & $15(88.2)$ & $4(100)$ \\
\hline PL7 & $0(0)$ & $1(5.9)$ & $0(0)$ \\
\hline PL12 & $1(25.0)$ & $1(5.9)$ & $0(0)$ \\
\hline Ro52 & $3(75.0)$ & $14(82.4)$ & $2(50.0)$ \\
\hline RF positive & $1(25.0)$ & $4(23.5)$ & $1(25.0)$ \\
\hline CK increase & $2(50.0)$ & $11(64.7)$ & $4(100)$ \\
\hline Aldolase increase & $2(50.0)$ & $12(70.6)$ & $4(100)$ \\
\hline \multicolumn{4}{|l|}{$\begin{array}{l}\text { Functional respiratory tests: } \\
\text { mean(CI95\%) }\end{array}$} \\
\hline Variation DLCOc(\%) & $-4.4(-12.5,3.7)$ & $-2.5(-6.1,1.0)$ & $7.4(-10.6,25.3)$ \\
\hline Variation FVC(\%) & $-1.1(-12.8,9.1)$ & $6.0(3.2,8.8)$ & $7.5(-9.5,24.6)$ \\
\hline Variation FEV1(\%) & $0(0-2.25)$ & $5.0(1.9,8.3)$ & $7.35(-18.0,32.7)$ \\
\hline Variation FEV1/FVC(\%) & $0.5(-7.1,8.2)$ & $-1.3(-2.22,-0.37)$ & $-0.4(-12.88,11.98)$ \\
\hline
\end{tabular}

Conclusion: ILD is a frequent and serious manifestation that can occur in patients with antisynthetase syndrome, which may have different radiological patterns. In our series, the most observed radiological pattern has been the NSIP pattern with $68 \%$. The pattern most associated with the Ro 52 antibody was the UIP pattern and the NSIP pattern. As for the group that required the largest numbers of drugs (including biological therapy) it was the NSIP pattern. When evaluating changes in respiratory function tests, the pattern that shows a tendency to improve over time is organized pneumonia with improvement of the DLCO, FVC, FEV1, FEV1 / FVC, while the UIP pattern showed a worsening of the DLCO and the FVC.

Disclosure of Interests: None declared

DOI: 10.1136/annrheumdis-2020-eular.3584

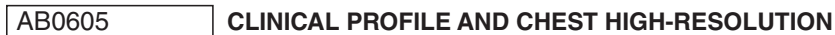 COMPUTED TOMOGRAPHY (HRCT) FINDINGS IN PATIENTS WITH CONNECTIVE TISSUE DISEASES AND INTERSTITIAL LUNG DISEASE: EXPERIENCE OF A SINGLE REFERENCE RHEUMATOLOGY CENTER}

$\underline{\text { I. Rusu }}^{1}$, L. Muntean ${ }^{1,2}$, M. M. Tamas ${ }^{1,2}$, I. Felea ${ }^{2}$, L. Damian ${ }^{1}$, I. Filipescu ${ }^{1,2}$, S. P. Simon ${ }^{1,2}$, C. Pamfil ${ }^{2}$, C. M. Marinescu ${ }^{3}$, M. A. Man ${ }^{2,4}$, C. Csutak ${ }^{2,3}$, S. Rednic ${ }^{1,2}$. $^{1}$ Emergency Clinical County Hospital, Rheumatology, ClujNapoca, Romania; "'luliu Hatieganu" University of Medicine and Pharmacy, Cluj-Napoca, Romania; ${ }^{3}$ Emergency Clinical County Hospital, Radiology, 